\title{
Alignment of Viewing-Angle Dependent Ultrasound Images ${ }^{\star}$
}

\author{
Christian Wachinger and Nassir Navab \\ Computer Aided Medical Procedures (CAMP), TUM, Munich, Germany \\ $\{$ wachinge, navab\}@cs.tum. edu
}

\begin{abstract}
We address the problem of the viewing-angle dependency of ultrasound images for registration. The reflected signal from large scale tissue boundaries is dependent on the incident angle of the beam. This applies an implicit weighting on the ultrasound image, dependent on the viewing-angle, which negatively affects the registration process, especially when utilizing curved linear transducers. We show that a simple reweighting of the images, considering a common physical model for ultrasound imaging, is not feasible. We therefore introduce a new matching function, separating reflectivity and scattering regions, which are the results of two different types of physical interactions of the ultrasound beam with the tissue. We use the local phase for identifying regions of reflectivity, and consider it as one part of our matching function, combining feature- and intensity-based aspects. First experiments provide good results for this novel registration approach.
\end{abstract}

\section{Introduction}

The fusion of several ultrasound images for the creation of a combined image with a larger field-of-view, referred to as ultrasound stitching, mosaicing or panorama imaging, is of clinical interest [1] and topic of on-going research. In the past, a variety of methods were proposed ranging from rigid to deformable [2, from intensity- to feature-based [3, and from pair- to groupwise [4]. While featurebased approaches may be an option for getting close to the correct alignment, the final alignment is best achieved with groupwise intensity-based methods 4 .

The major drawback of intensity-based methods is the missing overlap invariance of the similarity measures, favoring a total overlap of the images [4. The reasons for this phenomenon have not yet been investigated. They could either be rooted in the similarity measures themselves, like Cahill et al. [5] addressed, or in the ultrasound images. We show that the viewing-angle dependency of ultrasound images not only causes angle dependent artifacts like shadow, but also puts an implicit weighting on the images, favoring a total overlap.

To address this issue we first evaluate methods for reweighting ultrasound images, making them viewing-angle independent. However, currently used ultrasonic imaging models are not accurate enough, as we will show, for allowing

\footnotetext{
* This work was partly funded by the European Project "PASSPORT", ref. number 223904. The authors thank Andreas Keil and Reviewer 1 for valuable contributions.
} 
such a reweighting. We instead propose a new ultrasound matching function, consisting of one term, measuring the alignment of reflectivity structures, and a second term, measuring the alignment of the remaining texture. This new matching function has advantages for US-US, as well as, US-CT registration.

\section{Viewing Angle Dependency}

Structures, visible in ultrasound images, are the result of two different types of interaction of the ultrasound beam with the tissue: reflection and scattering. Reflection occurs at large scale tissue boundaries and is viewing-angle dependent. Scattering is caused by microscopic tissue inhomogeneities and provides the internal texture of the organs. Scattering is to a certain extent dependent on the direction of insonification because the ultrasound PSFs are not spherically symmetric. However, since we are not able to match single scattering responses from the resolution cells, it is more appropriate to consider entire scattering regions, and the echogeneity of these regions does not change with the viewing-angle.

A common physical model for the reflection, as it is described in the textbooks of Hedrick et al. [6] or Zagzebski [7, and further used by Wein et al. [8, is to calculate the reflected signal $R(\mathbf{x})$ as

$$
R(\mathbf{x})=\rho(\mathbf{x}) \cdot I_{i}(\mathbf{x}) \cdot(\cos \phi(\mathbf{x}))^{m}
$$

with the incident angle $\phi(\mathbf{x})$ at position $\mathbf{x}$, and the incident intensity $I_{i}$. The reflection coefficient calculates as

$$
\rho(\mathbf{x})=\left(\frac{z(\mathbf{x})-z(\mathbf{x}-\Delta \mathbf{d})}{z(\mathbf{x})+z(\mathbf{x}-\Delta \mathbf{d})}\right)^{2}
$$

with the acoustic impedance $z$ and $\Delta \mathbf{d}$ the distance between scan line points. The exponent $m$ models the heterogeneity of the tissue interface, ranging from rather specular reflections to diffuse reflectors. Typically, strong reflections can be observed at the diaphragm and the kidney surface.

To illustrate the problems, that affect the registration process when working with viewing-angle dependent ultrasound images, we create two artificial images, acquired from a curved linear probe, see Figure 1. The images show a region of high reflectivity, having a cosine intensity profile, and a scattering region. Rayleigh distributed noise is added to the images (log-compressed) to simulate the speckle noise of the ultrasound images 9]. The correct alignment of the two images, which can be acquired with lateral displacement, is to overlap the scattering regions. However, as we show in the similarity plot of Figure 1 . similarity measures like $C D_{2}[9$ favor a total overlap of the images. The reason is the dominance of the reflectivity structure, leaving only a local minimum at the desired position.

\subsection{Reweighting Ultrasound Images}

A first attempt, when thinking about the problem, is to identify structures of high reflectivity, calculate the incidence angle of the beam, and use the ultrasonic 

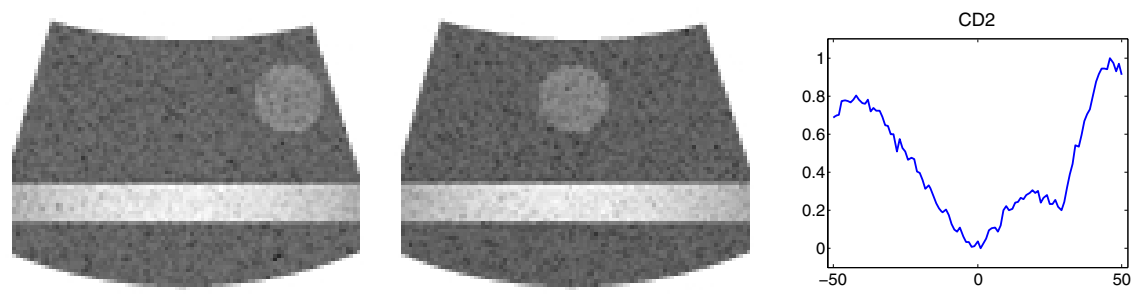

Fig. 1. Artificial ultrasound images showing reflection, scatter, and speckle. Similarity plot using $C D_{2}$, ] along lateral direction

imaging model as shown in Equation (11) to reweight the image. The result would be the creation of normalized ultrasound images being less or not at all viewing angle dependent. In the case of our example, the reflectivity region would result in a constant similarity value for translations in lateral direction, so that the scattering region would dominate the registration process.

In the following, we will investigate if the presented ultrasound model is accurate enough for reweighting the images. We acquired ultrasound B-mode and RF images with a curved linear transducer in a water bath with just one object in it, having a diffuse surface, usually used for single wall calibration, see Figure 2. The reflection coefficient $\rho(\mathbf{x})$ is the same for every beam, as we have the common water-object boundary. The incident intensity $I_{i}$ is dependent on the attenuation of the beam along its way to the boundary. The attenuation coefficient, which includes absorption and scattering effects, is $2.2 \cdot 10^{-3} \frac{\mathrm{dB}}{\mathrm{cm} \cdot \mathrm{MHz}}$ for water. In comparison, the attenuation coefficients for Fat $\left(0.6 \frac{\mathrm{dB}}{\mathrm{cm} \cdot \mathrm{MHz}}\right)$, Liver $\left(0.9 \frac{\mathrm{dB}}{\mathrm{cm} \cdot \mathrm{MHz}}\right)$, and Kidney $\left(1.0 \frac{\mathrm{dB}}{\mathrm{cm} \cdot \mathrm{MHz}}\right)$, are about three orders of magnitude larger. Further, the length of the beam in the middle and the one on the side vary about $2 \mathrm{~cm}(1.93$ exactly). This leads to an attenuation of $22.8 \cdot 10^{-3} \mathrm{~dB}$ by using an acquisition frequency of $2.6 \mathrm{MHz}$ and considering also the way back to the transducer. Since the ratio of change of intensity $\frac{I_{1}}{I_{0}}$ is close to 1 for values close to $0 \mathrm{~dB}$, we can neglect the effect of attenuation for our experiment.

The received signal, therefore only depends on the incident angle of the beam. The subsequent cost function has to be minimized in order to find the optimal exponent $m$, characterizing best the type of the interface

$$
\begin{aligned}
\mathcal{C}(m) & =\int\left(\frac{|R(\mathbf{x})|}{\cos ^{m}(\phi(\mathbf{x}))}-\rho(\mathbf{x}) \cdot I_{i}(\mathbf{x})\right)^{2} d \mathbf{x} \\
\frac{d \mathcal{C}(m)}{d m} & =\int 2\left(\frac{|R(\mathbf{x})|}{\cos ^{m}(\phi(\mathbf{x}))}-\rho(\mathbf{x}) I_{i}(\mathbf{x})\right)|R(\mathbf{x})| \cdot \ln (\cos (\phi(\mathbf{x}))) \cdot \cos ^{-m}(\phi(\mathbf{x})) d \mathbf{x}
\end{aligned}
$$

with $\rho(\mathbf{x}) \cdot I_{i}(\mathbf{x})$ being constant for all $\mathbf{x}$ on the surface and corresponding to the measured reflectivity of the middle beam, and $|R(\mathbf{x})|$ the absolute value of the received signal.

We plot the cost function in Figure 2(b), where a gradient descent optimization with the above stated gradient of the cost function leads to $m=34.52$. In Figure 2(c), we show the intensity profile of the RF data, together with 


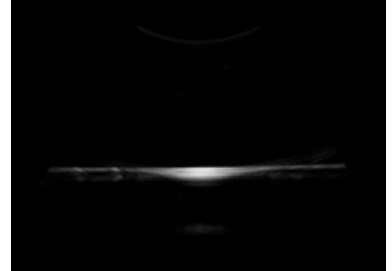

(a) B-Mode US image

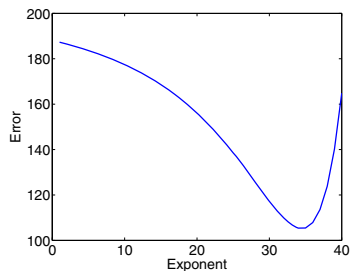

(b) Cost Function

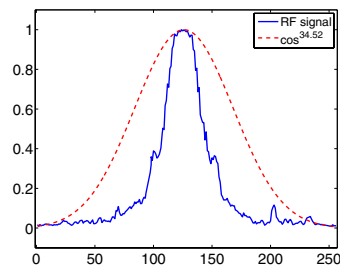

(c) Intensity Profile and Best Approximation

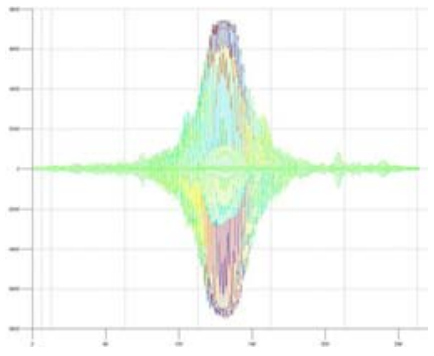

(d) RF Profile (x-axis: lateral)

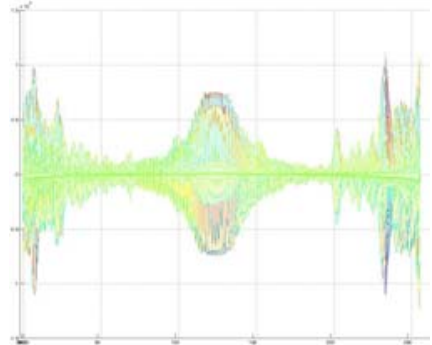

(e) Reweighted RF Profile

Fig. 2. Reweighting experiment on ultrasound RF data

the optimal cosine variant. The advantage of working with $\mathrm{RF}$ data is that no further processing steps or filters are applied on the data, making a meaningful evaluation of the model possible. When working with B-mode images, especially the log-compression has to be considered, turning the multiplicative cosine term of Equation (1) into an additive one. In Figure 2(e), we show the reweighted RF signal with $\cos ^{34.52}$. As can be seen, the result is not satisfying because instead of having a constant intensity profile, we have a "W" shaped one. For other interfaces it may be possible to find an exponent $m$ so that reweighting works, however, this would only characterize this specific type of interface and not be generally applicable. We conclude that the presented ultrasonic imaging model in Equation (11) is usually not accurate enough to allow for reweighting ultrasound images, in order to make them viewing-angle independent.

This finding also affects a recently introduced algorithm for US-CT registration [8, which bases upon the simulation of ultrasound images, applying this ultrasonic imaging model. The the next section, we introduce a new ultrasound matching scheme, usable for US-US and US-CT registration, to address this issue.

\section{A New Ultrasound Matching Scheme}

In this section we present a novel matching scheme for ultrasound images, dealing with the viewing angle dependency, discussed in the last section. As we 
concluded, a correction of the angle dependency of the images is with the current model not feasible. Our approach is therefore to separate reflection and scatter, which are the results of two different physical interactions of the beam with the tissue, combined in one image. In the following, we first present the method that we use for the crucial identification of regions of reflectivity in the ultrasound images, and then detail the new matching function.

\subsection{Reflectivity Regions}

We use local phase information for identifying regions of reflectivity because it provides us with structural information independent of the brightness and contrast [10]. This independence is very important for the extraction of reflectivity regions, since the absolute intensity of the reflection varies with the incident angle. The local phase was already used by Mulet-Prada and Noble [11] for boundary detection in echocardiography. Recently, Hacihaliloglu et al. [12] achieved good results in segmenting bone in ultrasound images employing the local phase. Mellor and Brady [13] apply mutual information on local phase images for multi-modal image registration. Grau et al. [10] use the local phase for aligning 3D echocardiographic sequences.

For 1-D signals the phase is constructed from the original signal and its Hilbert transform. Different approaches exist to extend this concept to $N$-D. In the following, we use the monogenic signal introduced by Felsberg et al. 14. It uses a generalization of the Hilbert transform, the Riesz transform, to calculate phase information in $N$-D. The image is filtered by $N$ filters, which are given in the Fourier domain by

$$
H_{i}\left(f_{1}, \ldots, f_{N}\right)=\frac{f_{i}}{\sqrt{\sum_{j=1}^{N} f_{j}^{2}}}
$$

with $f_{1}, \ldots, f_{N}$ the Fourier domain coordinates. We follow [10] in applying logGabor filters prior to the calculation of the monogenic signal of the image to extract frequency and spatial localization. The monogenic signal provides us with information about the local phase $P(x)$ each pixel $x$. We threshold the phase image with a value $\tau$ to obtain a binary mask, indicating reflectivity regions. Throughout all the experiments we use $\tau=0.7$, showing that this is not a crucial parameter. Alternatively, the local phase values could directly be used as weights, resulting in a fuzzy like mask.

\subsection{Matching Function}

Since the local phase image provides us with structural information independent of brightness, building a matching function upon this like in [10] would already significantly reduce the effects of the viewing-angle dependency on the registration. However, this also leads to the following drawbacks: First, we would be completely dependent on the outcome of the algorithm calculating the local phase. And second, texture information in the form of scattering regions is not integrated. Focusing on boundaries seems totally fine for echcardiographic 
applications, where not much texturing exists, but for other applications, like abdominal ones, this is not optimal. Consequently, the matching function we propose considers both, feature- and intensity-based aspects, by using reflection and texture.

Considering two images $u$ and $v$, an image grid $\Omega$, regions of reflectivity $\Gamma_{u}=\left\{x \in \Omega \mid P_{u}(x)>\tau\right\}$ and $\Gamma_{v}=\left\{x \in \Omega \mid P_{v}(x)>\tau\right\}$, further $\Gamma=\Gamma_{u} \cup \Gamma_{v}$, the matching function is

$$
\mathcal{M}(u, v)=\mathcal{M}_{\Omega \backslash \Gamma}^{T}(u, v)+\lambda \cdot \mathcal{M}_{\Omega}^{R}\left(P_{u}, P_{v}\right)
$$

with $\mathcal{M}_{\Omega \backslash \Gamma}^{T}$ measuring the similarity of the texture regions, excluding reflectivity regions $\Omega \backslash \Gamma$, and $\mathcal{M}_{\Omega}^{R}\left(P_{u}, P_{v}\right)$ measuring the similarity of the phase images on the whole grid $\Omega$. The matching function can be easily extended to the multivariate case by using the multivariate similarity framework proposed in [4], summing up pairwise terms.

Suitable similarity measures for $\mathcal{M}^{T}$ are, next to standard measures like sum of squared differences (SSD), normalized cross-correlation (NCC), and mutual information (MI) assuming a Gaussian distributed noise, the similarity measures proposed by Cohen and Dinstein 9, assuming a Rayleigh distributed noise. Especially $C D_{2}$ was reported to have good performance [15. Suitable similarity measures for $\mathcal{M}^{R}$ are once again the standard measures, and the similarity measure proposed in [10, taking the cosine of the phase difference.

The disadvantage, when choosing two different similarity measures, is that the correct selection of the weighting term $\lambda$ is important, since it has to map the similarity score into a comparable range. Even when working with normalized images, the outcomes of the similarity measures are not directly comparable, so that we perform the experiments with the same similarity measures for both terms and simply set $\lambda=1$.

This matching framework is also directly applicable to the US-CT registration 8], where texture and reflection parts are simulated separately and finally combined during the registration. In our matching approach, reflectivity and texture regions are extracted from the ultrasound image, and can directly be matched with the simulated ones.

\section{Experiments}

In Figure 3. we show the results of the new matching function, using SSD as similarity metric for texture and reflection, for the data set illustrated in Figure 1. Comparing the similarity plots for $C D_{2}$ and $\mathcal{M}^{T}$, which both use the texture, we can see the improvement of not considering the reflectivity region $\Gamma$ in the calculation. The plot of the term $\mathcal{M}^{R}$, measuring the similarity of the phase images is very smooth, however, does not indicate the correct alignment at 30 . Combining both curves, $\mathcal{M}$, leads to an accurate cost function with wider capture range than $\mathcal{M}^{T}$. 


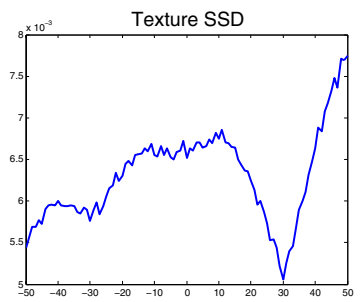

(a) $\mathcal{M}^{T}$

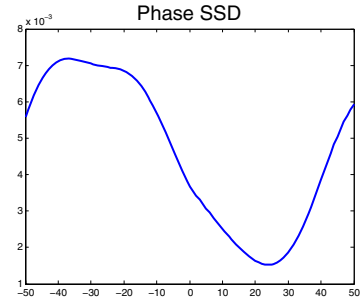

(b) $\mathcal{M}^{R}$

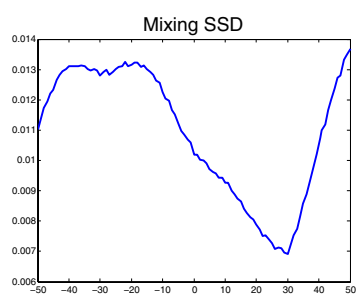

(c) $\mathcal{M}$

Fig. 3. Similarity plots for images from Figure 1 along lateral direction using SSD

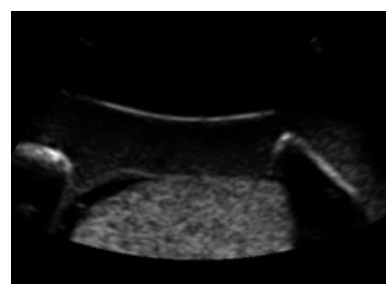

Texture NCC

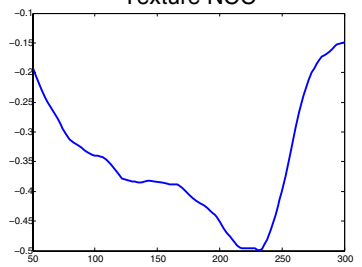

(d) $\mathcal{M}^{T}$

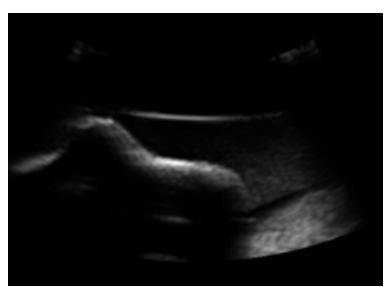

Phase NCC

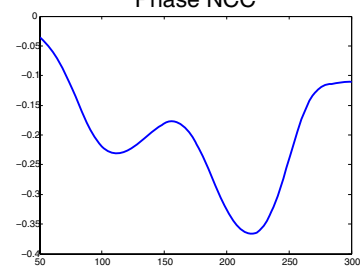

(e) $\mathcal{M}^{R}$

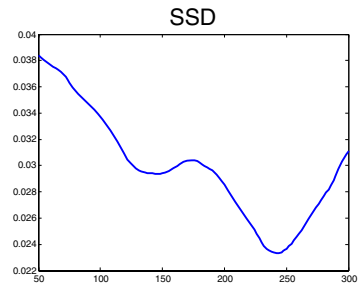

Mixing NCC

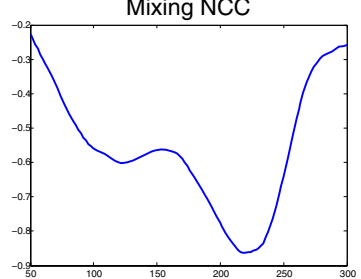

(f) $\mathcal{M}$

Fig. 4. Ultrasound images acquired from laterally displace positions. Similarity plots for SSD and the different parts from our new matching scheme.

We also conducted experiments for US-US registration on ultrasound images acquired with a curved linear transducer, see Figure 4. The images are acquired from an ultrasound phantom, with a true displacement of $88.0 \mathrm{~mm}$. The bony structure on the left side of the first image is depicted in the middle of the second image. The similarity plots for SSD, NCC, and $C D_{2}$ are comparable, with SSD indicating the correct alignment at $96.8 \mathrm{~mm}$. The combined cost function indicates the correct alignment at $88.8 \mathrm{~mm}$, with $\mathrm{NCC}$ as similarity measure for both terms.

We performed further experiments on a data set, similar to the one presented by Penney et al. 16, simulating the US-CT registration problem. An illustration of the images together with the good results of our matching approach can be found in the supplementary material 1 .

${ }^{1}$ http://www.webcitation.org/5hEZWaajc 


\section{Conclusion}

The alignment of ultrasound images is complicated by the viewing-angle dependent nature of the images. After an analysis of the effects on the registration process, we presented a method for reweighting ultrasound images. However, the commonly applied imaging model for ultrasound was shown to be not accurate enough, for allowing a correction of the viewing-angle dependency. Instead, we introduced a new matching scheme for the alignment of ultrasound images, which can be used for US-US as well as US-CT registration. It incorporates the usage of feature- and intensity-based metrics into one global measure. We evaluated the new registration approach on various data sets, with good results.

\section{References}

1. Henrich, W., Schmider, A., Kjos, S., Tutschek, B., Dudenhausen, J.W.: Advantages of and applications for extended field-of-view ultrasound in obstetrics. Archives of Gynecology and Obstetrics 268, 121-127 (2003)

2. Poon, T., Rohling, R.: Three-dimensional extended field-of-view ultrasound. Ultrasound in Medicine and Biology 32(3), 357-369 (2005)

3. Ni, D., Qu, Y., Yang, X., Chui, Y.-P., Wong, T.-T., Ho, S.S.M., Heng, P.-A.: Volumetric ultrasound panorama based on 3D SIFT. In: Metaxas, D., Axel, L., Fichtinger, G., Székely, G. (eds.) MICCAI 2008, Part II. LNCS, vol. 5242, pp. 52-60. Springer, Heidelberg (2008)

4. Wachinger, C., Wein, W., Navab, N.: Three-dimensional ultrasound mosaicing. In: Ayache, N., Ourselin, S., Maeder, A. (eds.) MICCAI 2007, Part II. LNCS, vol. 4792, pp. 327-335. Springer, Heidelberg (2007)

5. Cahill, N., Schnabel, J., Noble, J., Hawkes, D.: Revisiting overlap invariance in medical image alignment. In: MMBIA (2008)

6. Hedrick, W.R., Hykes, D.L., Starchman, D.E.: Ultrasound Physics and Instrumentation. Mosby, 4th edn. (2004)

7. Zagzebski, J.: Essentials Of Ultrasound Physics, 1st edn., Mosby (1996)

8. Wein, W., Brunke, S., Khamene, A., Callstrom, M.R., Navab, N.: Automatic ctultrasound registration for diagnostic imaging and image-guided intervention. Medical Image Analysis 12, 577-585 (2008)

9. Cohen, B., Dinstein, I.: New maximum likelihood motion estimation schemes for noisy ultrasound images. Pattern Recognition 35(2), 455-463 (2002)

10. Grau, V., Becher, H., Noble, J.: Registration of multiview real-time 3-d echocardiographic sequences. IEEE Transactions on Medical Imaging 26(9) (September 2007)

11. Mulet-Parada, M., Noble, J.: 2D+ T acoustic boundary detection in echocardiography. Medical Image Analysis 4(1), 21-30 (2000)

12. Hacihaliloglu, I., Abugharbieh, R., Hodgson, A.J., Rohling, R.: Bone segmentation and fracture detection in ultrasound using 3D local phase features. In: Metaxas, D., Axel, L., Fichtinger, G., Székely, G. (eds.) MICCAI 2008, Part I. LNCS, vol. 5241, pp. 287-295. Springer, Heidelberg (2008)

13. Mellor, M., Brady, M.: Phase mutual information as a similarity measure for registration. Medical Image Analysis 9(4), 330-343 (2005)

14. Felsberg, M., Sommer, G.: The monogenic signal. IEEE Transactions on Signal Processing 49(12), 3136-3144 (2001)

15. Boukerroui, D., Noble, J.A., Brady, M.: Velocity estimation in ultrasound images: a block matching approach.. Inf. Process Med. Imaging 18, 586-598 (2003)

16. Penney, G., Griffin, L., King, A., Hawkes, D.: A novel framework for multi-modal intensity-based similarity measures based on internal similarity. SPIE 6914 (2008) 